\title{
Laryngotracheal Stenosis: Clinical Aspects and Management Challenges
}

\author{
Meryem Lahjaouj ${ }^{1}$ Khadija El Bouhmadi ${ }^{1}$ Youssef Oukessou ${ }^{1} \quad$ Sami Rouadi ${ }^{1}$ Reda Abada ${ }^{1}$ \\ Mohamed Roubal ${ }^{1}$ Mohamed Mahtar ${ }^{1}$
}

1Department of Otolaryngology and Head and Neck Surgery, 20 August Hospital, Casablanca, Morocco

\begin{abstract}
Address for correspondence Khadija El Bouhmadi, MD, Department of Otorhinolaryngology and Head and Neck Surgery, 20 August Hospital, Casablanca 20360, Morocco (e-mail: elbkhadija25@gmail.com).
\end{abstract}

Int J Recent Surg Med Sci 2021;7:17-22.

\begin{abstract}
Laryngotracheal stenosis (LTS) is a severe condition with a high morbidity rate, described as the narrowing of the upper airway diameter. The etiology of acquired LTS is dominated by prolonged tracheal intubation. The diagnosis is based on endoscopic examination and computed tomography scan imaging providing an exact analysis of the lesion, with precise measurements.

We report a descriptive retrospective review based on the analysis of the medical records of a cohort of 18 patients, treated between January 2015 and January 2018 for an acquired LTS.

The results showed predominance in young males. The majority (73\%) was hospitalized in a reanimation unit under tracheal intubation while the others $(27 \%)$ underwent a tracheostomy. The location of the stenosis was tracheal in $69 \%$ of the patients, laryngeal in $23 \%$, and laryngotracheal in $8 \%$, and the degree of obstruction exceeded $70 \%$ in $11.2 \%$. The endoscopic treatment was based on dilatation and endoluminal calibration by the Montgomery T-tube in $77.7 \%$ of our patients, circumferential resection by $\mathrm{CO}_{2}$ laser in $11.1 \%$, and endoscopic posterior cordectomy in $11.1 \%$ of the cases. The surgical treatment relied on the resection of the stenosis followed by T-tube calibration in $61.1 \%$ of our patients, the resection with a termino-terminal anastomosis in $11 \%$, and supraglottic partial laryngectomy in one patient. The follow-up showed

Keywords

- laryngotracheal stenosis

- Montgomery T-tube

- laryngotracheal reconstruction major improvement in the clinical symptomatology, but the biggest challenge in LTS remains the occurrence of restenosis.

LTS is a severe condition and needs to be always considered in patients with inspiratory dyspnea and a medical history of tracheal intubation. It requires exhaustive endoscopic and imaging exploration with multidisciplinary management and long-term follow-up to prevent and detect the early signs of an eventual recurrence.
\end{abstract}

\section{Introduction}

Laryngotracheal stenosis (LTS) is a severe condition described as a narrowing of the upper airway between the larynx and the trachea with potentially devastating consequences. The

published online Feburary 10, 2021
DOI https://doi.org/

$10.1055 / \mathrm{s}-0041-1723923$ ISSN 2455-7420. origin can be congenital or, most frequently, acquired as a result of trauma, related to endotracheal intubation due to external traumas, and tracheotomies, or due to a neoplasm, autoimmune, or infectious process. ${ }^{1}$ (c) 2021. Medical and Surgical Update Society.

This is an open access article published by Thieme under the terms of the Creative Commons Attribution-NonDerivative-NonCommercial-License, permitting copying and reproduction so long as the original work is given appropriate credit. Contents may not be used for commercial purposes, or adapted, remixed, transformed or built upon. (https://creativecommons.org/licenses/by-nc-nd/4.0/). Thieme Medical and Scientific Publishers Pvt. Ltd. A-12, 2nd Floor, Sector 2, Noida-201301 UP, India 
The clinical presentation is dominated by dyspnea, or respiratory failure in the advanced forms, requiring a tracheotomy as a saving procedure. The imaging and endoscopic exploration is very contributory in specifying the stenosis characteristics. The optimal management of LTS needs a multidisciplinary approach. Besides, next to endoscopic expansion dilatation and Montgomery T-tube tracheal stent calibration, $\mathrm{CO}_{2}$ laser and open surgery resection, in addition to partial laryngectomy and laryngotracheoplasty, offer multiple therapeutic options depending on the site, the type, and the measurements of the stenosis. However, the risk of recurrences still high, the restenosis often occurs, leading to an iatrogenic vicious circle where the patients lose their laryngotracheal functions majorly affecting their quality of life. ${ }^{2}$

The aim of our work is to describe the clinical aspects, the imaging and endoscopic findings, and the treatment modalities of LTS; and to analyze the results of operated patients, exposing the management difficulties of this particular condition.

\section{Patients and Methods}

We performed a descriptive retrospective review based on the analysis of the medical records of a cohort of 18 patients, treated at the Department of Head and Neck Surgery of the 20 August Hospital of Casablanca, between January 2015 and January 2018, for an acquired nontumoral laryngeal, tracheal, or laryngotracheal stenoses. All other etiologic forms of stenosis were excluded.

The medical history and epidemiological parameters were stated. The causal factors of the occurrence of the LTS were highlighted with a description of the first revealing symptoms and the initial medical care required. Then, the clinical, radiological, and endoscopic data of all the patients were completed and compared to assess the different stenoses parameters. The treatment varied but all the patients underwent a surgical procedure, the details of which were reported.

The postoperative follow-up was assured on short- and long term, looking for any sign of recurrence. The restenosis has been assessed in terms of time limit, clinical aspect, location and degree of obstruction, and the chosen curative treatment.

The qualitative variables were analyzed in frequency and percentage, while the quantitative variables were analyzed in medians.

\section{Results}

Epidemiological Features and Medical History

Among the 18 patients collated, there were 14 males (77\%) and 4 females (23\%), with a sex ratio up to 3.5 . The median age was 26.7 years (19-59 years) with a high majority of young people under 30 years of age representing $77 \%$ ( - Fig. 1 ).

No particular medical history was noted except a total thyroidectomy for three of the patients.

The stay in reanimation unit was observed in 13 patients (73\% of cases) secondary to severe cranial trauma in

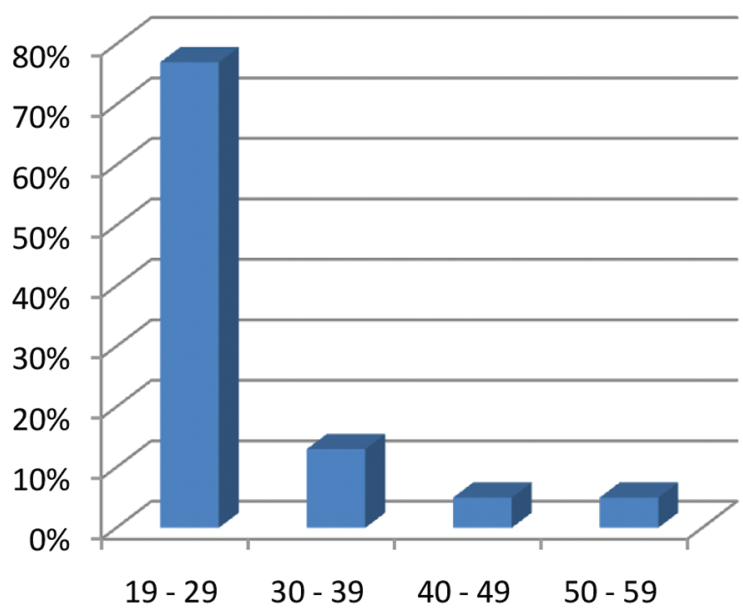

Fig. 1 Distribution of patients according to age.

10 patients (55\%), severe pneumonia in 2 patients (13\%), and Guillain-Barré syndrome in 1 patient (5\%). Regarding the prognosis and the need of prolonged endotracheal intubation, $30 \%$ of these patients underwent a preventive and programmed tracheotomy after 5 to 30 days, with a median of 20 days.

The five patients (27\%) with no history of tracheal intubation required a tracheotomy for bilateral paralysis of the two vocal cords, secondary to bilateral surgical lesion of the two recurrent laryngeal nerves during total thyroidectomy 3 to 4 months ago in two cases and external laryngeal trauma in three cases to secure the upper airway.

The follow-up showed that the $43 \%$ of our patients who did not undergo a preventive tracheotomy in the reanimation unit presented a respiratory distress immediately after extubation or days later after discharge with an average free interval of 2 weeks and then, all needed a rescue tracheotomy. Also, the other 30\%, despite their preventive tracheotomy, presented inspiratory dyspnea on an average of 3 weeks after their decannulation, requiring the replacement of the tube. At last, the two patients with bilateral vocal cord paralysis were treated surgically for a laser cordectomy and the three patients with laryngeal trauma were kept under observation for 1 month and they all presented inspiratory dyspnea after the removal of the tracheotomy tube requiring another tracheotomy.

Thus, tracheotomy was finally done onto the totality of our patients, either as a preventive and planned procedure in $30 \%$ of the cases in the reanimation unit, or as a saving procedure in front of acute respiratory distress, most frequently in the emergency room, in $70 \%$ of the cases.

\section{Clinical Features}

The main symptom was inspiratory dyspnea as found in 70\% of our patients, possibly associated with dysphonia, immediate or progressive, possibly leading to respiratory failure.

The clinical examination of the patients admitted to the emergency room, with a history of tracheal intubation, 
laryngeal trauma, or total thyroidectomy, found conscious patients, Glasgow Coma Scale score up to 15, cyanotic with stridor, and suprasternal retraction. A scar of thyroidectomy was found in two patients.

\section{Clinical and Endoscopic Exploration}

The clinical and endoscopic exploration is based on flexible rhino-laryngoscopy and direct rigid laryngoscopy allowing the precise analysis of the LTS parameters.

- Site: The location of the stenosis was tracheal in $69 \%$ of the patients, laryngeal in $23 \%$, and laryngotracheal in $8 \%$.

- Subsite: For the strictly laryngeal site, the stenosis was supraglottic in one patient, glottic in one patient, and subglottic in the last one.

- Aspect: An inflammatory aspect of the stenosis was found in all the patients.

- Degree of obstruction: The reduction in the laryngeal or tracheal lumen was evaluated between 0 and $50 \%$ in 10 patients (55.5\%), between 51 and $70 \%$ in 6 patients (33.3\%), and between 71 and $100 \%$ in 2 patients (11.2\%; -Fig. 2).

The mobility of the larynx was preserved in 16 patients and reduced in 2 .

\section{Radiological Data}

A front chest X-ray was done in all of our patients showing the LTS in only five cases (28\%) as a narrowing of the tracheal clarity regarding the seventh cervical vertebrae.

The cervical computed tomography (CT) scan remains the most contributory imaging examination for a more precise assessment of the stenosis. It was done in $76.9 \%$ of our patients, showing the stenosis as a circumferential thickening or a narrowing of the laryngotracheal lumen. In 53.8\%, exact measurements of the stenosis were given, always exceeding $1 \mathrm{~cm}$, up to $5 \mathrm{~cm}$ in one patient ( - Fig. 3 ).

\section{Therapeutic Management}

The medical treatment was indicated to all patients and started with oral corticotherapy in a dose of $1 \mathrm{mg} / \mathrm{kg}$ per day for 5 to 10 days. Antibiotics were reserved for patients with

\section{$0-50 \% \square 51-70 \% \square 71-100 \%$}

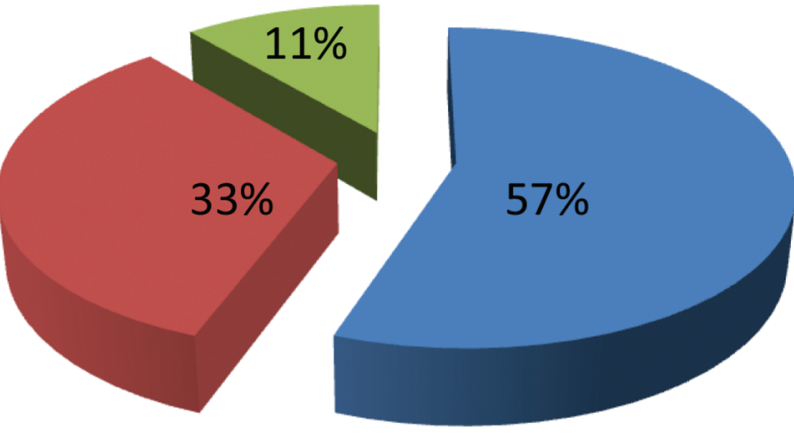

Fig. 2 Distribution by degree of stenosis obstruction.

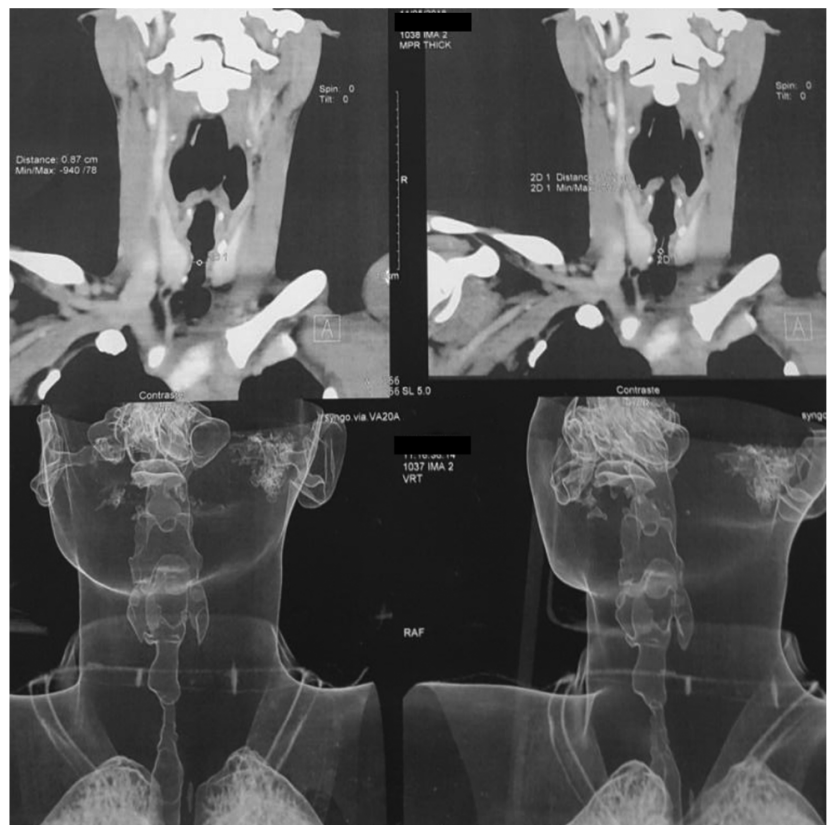

Fig. 3 Computed tomography scan imaging of a laryngotracheal stenosis.

signs of infection based on amoxicillin/clavulanic acid in a dose of $150 \mathrm{mg} / \mathrm{kg}$ per day for 8 days.

The endoscopic treatment based on dilatation by adapted expansive candle and endoluminal calibration by the Montgomery T-tube tracheal stent was performed in the majority of our patients (77.7\%). The circumferential resection of the LTS by $\mathrm{CO}_{2}$ laser was done in $11.1 \%$ of the patients while an endoscopic posterior cordectomy was performed in $11.1 \%$ of the cases.

For the surgical treatment, different options were indicated. The majority of our patients (61.1\%) underwent a resection of the stenosis via an external approach followed by a calibration by the Montgomery T-tube. A resection of stenosis with a termino-terminal anastomosis was performed in $11 \%$ of the patients, while one patient was operated for a supraglottic partial laryngectomy.

\section{Evolution and Follow-Up}

The follow-up was conducted from the early postoperative stage and was based on clinical, endoscopic, and imaging exploration.

The short-term follow-up showed major clinical improvement with total disappearance of dyspnea, with no swallowing disorder. The hospital stay was short and no further complication was noted.

The medium-term follow-up was focused on the success of decannulation with a median time limit of 2 weeks, until 1 month for three patients (those operated for resection anastomosis and the supraglottic partial laryngectomy). On the other hand, the Montgomery T-tube was kept for 9 to 12 months.

The long-term follow-up was centered on the return to a normal functioning life and, mostly, to detect eventual recurrences. The evaluation of the therapeutic results was based clinically on the total absence of dyspnea during daily and 
professional activities. Endoscopic examination and cervical CT scan were done and showed major improvement in the initial LTS in $27.7 \%$ of the cases (after $\mathrm{CO}_{2}$ laser and T-tube treatments). On the other side, $55.5 \%$ of the cases developed a recurrence of the stenosis, in the same site, usually lower. The restenoses were treated initially by Montgomery T-tube.

\section{Discussion}

LTS is defined as a luminal narrowing of the larynx and/ or trachea secondary to pathological fibroinflammatory repair process. ${ }^{3}$ It is a high-morbidity condition, showing a rise in recent years because of increased incidence of prolonged intubation, tracheotomy for head and neck pathologies, and recovery of patients out of intensive care and reanimation units. ${ }^{4}$

The most concerned age range seems to be of 20 to 30 years, as it showed in our series and in Babu et al's study. ${ }^{2}$ This age range is also the most concerned by severe cranial trauma and polytrauma secondary to road traffic injuries, requiring a stay in the reanimation unit. A second peak is also reported at the fifth decade related to the recrudescence of medical affections. Thus, the majority of our patients with history of reanimation stay were either hospitalized for severe trauma or medical condition affecting the respiratory function.

For the same higher susceptibility for trauma, the masculine predominance found in our study (77\%) is also described by Cuisinier et $\mathrm{al}^{5}$ up to $65 \%$. However, Stauffer et $\mathrm{al}^{6}$ deny the positive correlation between masculine gender and LTS.

The physiopathology of the LTS is a mucosal ischemia and ulceration secondary mainly to the pressure of the intubation tube cuff. The inflammatory reaction and local infection lead to progressive damage of the cartilaginous body. The tracheotomy, by directly affecting the tracheal cartilaginous rings, is a supplementary factor for tracheal instability. So, LTS regroup retractile fibrosis of the mucosa and instability of the cartilaginous structure (malacia). ${ }^{7}$

Schematically, three types of tracheal stenosis were described in the literature. The short and simple stenosis $(<1 \mathrm{~cm})$ with a diaphragm shape is secondary to concentric and retractile fibrosis of the mucosa, usually sparing the cartilaginous rings. The complex stenosis $(\geq 1 \mathrm{~cm})$ with a tortuous shape affects the totality of the tracheal wall, specifically the cartilaginous rings. Tracheomalacia is frequently associated in this type, usually detected after the dilatation. And the pseudo-glottal stenosis with an A shape is secondary to a rupture of the tracheal cartilages after the tracheostomy inducing a short-portion tracheomalacia. ${ }^{7}$

- Table 1 summarizes the predisposing factors related to tracheal intubation and tracheostomy. 2,5

Anand et $\mathrm{al}^{8}$ reported an incidence of 0.6 to $21 \%$ of LTS after an intubation and an incidence of 6 to $21 \%$ after a tracheotomy. But the incidence can reach 20 to $30 \%$ when there is a succession of intubation/tracheotomy, increasing the duration of mechanical ventilation. ${ }^{9-11}$

The time limit when the laryngotracheal lesions appear is widely discussed. Our patients started developing lesions after 5 days. Keane et a ${ }^{11}$ consider the intubation harmless for 6 days while it is 8 days for Pontoppidan et al..$^{12}$ Another study showed that laryngotracheal lesions start in the first 17 hours after the trauma in adults and in the first week in children. ${ }^{2}$ On the other hand, Baugnée et $a{ }^{13}$ showed that also the very short intubation for less than 24 hours can cause secondary scar stenosis., ${ }^{2,13}$

Also, Sinha et $\mathrm{al}^{14}$ demonstrated significant mucosal injury following short-term tracheal intubation at near recommended cuff inflation pressures on animal model. For a duration of tracheal intubation of 191 minutes (standard deviation \pm 41.6 ), in all except one animal, cuff pressures were maintained in the range of 25 to $45 \mathrm{cmH}_{2} 0$. Histopathological findings showed more extensive changes than previously described, seen in all mucosal layers consistent with acute, suppurative, and ulcerative tracheitis.

Inspiratory dyspnea in a patient with a history of tracheal intubation or tracheotomy is the main clinical revealing symptom, found in almost $100 \%$ of the cases in literature. However, other signs should warn as dysphonia, exertional dyspnea, wheezing, shortness of breath to hoarseness, frank stridor, or bradypnea. ${ }^{15}$

The endoscopic exploration has a capital importance considering its contribution in the evaluation of the location, expanse and obstruction degree of the stenosis, and the state of the local mucosa. It also allows taking samples for biopsy.

The most common location in our study was the trachea with a rate of $69 \%$, followed by the larynx in $23 \%$ of the cases with an equal distribution of its three levels, then the LTS in $8 \%$ of the cases. However, Babu et al's study ${ }^{2}$ found a higher incidence of subglottic laryngeal stenosis with a rate up

Table 1 Predisposing factors of laryngotracheal stenosis related to intubation. ${ }^{2,5}$

\begin{tabular}{|c|c|c|c|c|}
\hline $\begin{array}{l}\text { Related to the } \\
\text { patient }\end{array}$ & Related to the tube & $\begin{array}{l}\text { Related to the } \\
\text { intubation }\end{array}$ & Related to care & $\begin{array}{c}\text { Related to } \\
\text { "tracheotomy" }\end{array}$ \\
\hline $\begin{array}{l}\text { - Children } \\
\text { - Women } \\
\text { - Tracheal affection: } \\
\text { malformation, } \\
\text { hypoplasia, infection } \\
\text { - Systemic affection: } \\
\text { immunosuppression, } \\
\text { hypoxia, anemia ... }\end{array}$ & $\begin{array}{l}\text { - Too large external } \\
\text { diameter } \\
\text { - Stiff consistency } \\
\text { - High pressure cuff } \\
\text { - Bad compatibility } \\
\text { - Continuous } \\
\text { movement of the tube } \\
\text { in the lumen }\end{array}$ & $\begin{array}{l}\text { - Traumatic } \\
\text { - Prolonged } \\
\text { - Sequence } \\
\text { intubation- } \\
\text { tracheostomy }\end{array}$ & $\begin{array}{l}\text { - Inadequate } \\
\text { sedation } \\
\text { - Nasogastric tube } \\
\text { - Assisted ventilation } \\
\text { - Traumatic and non- } \\
\text { sterile aspirations } \\
\text { - Insufficient air } \\
\text { humidification }\end{array}$ & $\begin{array}{l}\text { - Emergency } \\
\text { cricothyrotomy } \\
\text { - High level } \\
\text { tracheotomy }\end{array}$ \\
\hline
\end{tabular}


to $61.9 \%$, followed by the tracheal stenosis (33.3\%), then the LTS (4.7\%). Also, Zribi et al's ${ }^{16}$ study considered the superior third as the most affected in tracheal stenosis as long as it is the most injured by intubation tube.

Also, the length of the stenosis in all our patients was measured between 1 and $6 \mathrm{~cm}$, while the degree of obstruction was evaluated with a predominance of an obstruction under $50 \%$. The Zribi et al $^{16}$ study found a rate of $66.6 \%$ of stenosis from 2 to $4 \mathrm{~cm}, 28.5 \%$ below $2 \mathrm{~cm}$, and $4.7 \%$ above $4 \mathrm{~cm}$, with a median degree of obstruction at $75 \%$ (50-95\%); while Cuisinier et $\mathrm{al}^{5}$ reported the median degree of obstruction at $64 \%(10-95 \%)$.

The CT scan was done in $76.9 \%$ of cases in our study and remains the reference imaging examination. Actually, the CT scan multislice presents advantages such as reduction in respiratory and cardiac artifacts, absence of unexplored interval, and the precise measurements in the three space planes, in addition to reconstructions and dynamic explorations. ${ }^{17}$ Thus, multiple studies highlighted its high sensitivity and specificity in LTS stenosis, proposing it as a replacement of endoscopic exploration.

Management of LTS is challenging and requires a multidisciplinary approach. The fundamental aims of the treatment are airway patency, glottic competence for airway protection against aspiration, and acceptable voice quality, in that order of priority. ${ }^{2}$

The first step consists of medical treatment to reduce the local inflammation and stabilize the stenosing lesions based on corticosteroids, antibiotics, acid reflux treatment, and mitomycin C, even if a study by Madan et al concluded that mitomycin $\mathrm{C}$ is not an effective treatment for postintubation tracheal stenosis. ${ }^{18}$

If tracheotomy is the simplest treatment method, used in our study for patients with respiratory failure coming to the emergency, it has limitations like the inability to vocalize, the inaesthetic disfigurement, and the impossibility for certain activities such as swimming. ${ }^{2}$

The endoscopic management is indicated for lesion size $<1 \mathrm{~cm}$ without framework destruction and is based on dilatation of the stenosis with or without the use of electrocautery or laser for luminal enhancement, which has the advantage of precision, maneuverability, good hemostasis, and less postoperative edema, and can be used before or after surgery. With these measures, the relief is immediate and the result can be curative in some and provide an excellent bridge to surgical management in the rest. However, many reports show high recurrence. ${ }^{19}$ In Yamamoto et al's meta-analysis, ${ }^{20}$ success rates of endoscopic dilatation and laser resection (six articles) varied between 40 and $82 \%$, but when the indication was a lesion size $<1 \mathrm{~cm}$ without framework destruction, the results were significantly better than in patients with stenosis lengths $>1 \mathrm{~cm}$, showing the importance of the good indication.

Surgery remains the main curative treatment, but it is still difficult, needing rigorous postoperative nursing. The indications for laryngeal or laryngotracheal stenosis vary depending on the mobility of the arytenoid, the length of the stenosis, the state of the cricoid cartilage, and the degree of damage in the subglottic area, and they are summed up as follows: ${ }^{21}$

- In case of normal arytenoid mobility: If the stenosis is limited to the cricoid cartilage, an enlargement laryngotracheoplasty by a cartilage graft is performed, usually without calibration. If the stenosis is circular, an anterior and posterior cricotomy is done with the interposition of a cartilage fragment in the two incisions, plus a calibration by a Silastic sheet or a Teflon tube.

- In case of glottic immobility secondary to a cricoarytenoid ankylosis with severe and extended subglottic stenosis: It is possible to combine cricotracheal resection with posterior vertical cricotomy, cartilage interposition, and prolonged calibration. A laryngotracheoplasty with anterior and posterior enlargement associated to a quadrisection of the cricoid can also be adapted.

The type of the tracheal stenosis highly guides the surgical procedure. The inflammatory short and simple tracheal stenosis, after the medical treatment, is best managed by endoscopic interventions, such as dilatation by an expansion candle or endoluminal calibration. However, in case of complex stenosis concerning a long segment with cartilage destruction, surgery is the preferred modality with better long-term results, based on a resection of the affected portion followed by a termino-terminal anastomosis. The surgery should be cautiously done; the lateral tracheal dissection should be restricted only to the stenosed segment to avoid ischemia to the tracheal ends with good care on avoiding injury to the recurrent laryngeal nerves. ${ }^{19}$ In Yamamoto et al's meta-analysis, ${ }^{20}$ pooled success rate of laryngotracheal resection and anastomosis (12 articles) was 95\%.

Endotracheal prosthesis (stents) is recommended in benign tracheal stenosis only if the patient is unfit for, or refuses surgery, or as a bridge to surgery, and should not be used as a definitive treatment regarding its important tracheal damages. $^{22}$

Otherwise, Montgomery T-tube stays the best alternative when reconstructive surgery cannot be done. Working as a tracheal stent and a tracheostomy tube, it allows the preservation of voice, maintenance of nasal respiration, and a good tolerance with less local inflammatory reaction, in addition to being a socially acceptable device with easy daily care. ${ }^{23}$ Regarding the amount of time it should stay in place, Huang's study ${ }^{24}$ recommends a duration of 6 to 12 months, prolonged after revision. The results with the Montgomery T-tube are satisfying as the totality of our patients with the T-tube showed major clinical improvement with a disappearance of dyspnea for 3 years; results are confirmed by Dass et al's study ${ }^{25}$ on 71 patients concluding to a favorable result after treatment by T-tube in $91.5 \%$ of the cases, with a success of decannulation.

Our study reports a rate of restenosis up to $60 \%$, secondary to a granuloma with a lower stenosis. These recurrences were treated by the excision of the new lesion and the setting up of a Montgomery T-tube of a larger size. The Zribi et al study ${ }^{16}$ advises the use of the T-tube in first intention in association with other therapeutic modalities; specifying also its 
efficiency in the treatment of recurrences whose evolution and follow-up were favorable. Also, for the management of recurrences, endoscopic treatment can be used, or a second surgical procedure but with an interval of 1 year at least, to stabilize the stenosis.

\section{Conclusion}

LTS represents a particular condition with an increasing incidence and a high morbidity. The main causal factor still is the prolonged tracheal intubation. The clinical presentation is dominated by dyspnea.

The management of the LTS is very challenging. The resection-anastomosis is the treatment of choice even though its feasibility is difficult when the stenosis concerns the glottis or subglottis. Otherwise, the Montgomery T-tube seems more efficient and should be always considered according to its effectiveness on airway permeability and voice restoration.

The main complication of LTS is the high incidence of recurrence that can occur multiple times affecting sorely the anatomical function and the patient's quality of life.

\section{Funding}

None.

\section{Conflict of interest}

None declared.

\section{References}

1 Almanzar A, Danckers M, Laryngotracheal Stenosis. Treasure Island, FL: StatPearls Publishing; 2020

2 Babu MM, Kumar RA, Thirugnanamani R. Montgomery T-tube for management of tracheal stenosis: a retrospective analysis in a government hospital of South India. Int J Phonosurg Laryngol 2016;6(2):73-77

3 Woliansky J, Paddle P, Phyland D. Laryngotracheal stenosis management: a 16-year experience. Ear Nose Throat J 2019. doi: $10.1177 / 0145561319873593$

4 Montgomery WW. T-tube tracheal stent. Arch Otolaryngol 1965;82:320-321

5 Cuisinier O, Righini C, Pison C, Ferreti G. Surgical and/or endoscopic treatment of acquired tracheal stenosis in adult patients. Ann Otoloaryngol Chir Cervicofac 2004;121:3-13

6 Stauffer JL, Olson DE, Petty TL. Complications and consequences of endotracheal intubation and tracheotomy. A prospective study of 150 critically ill adult patients. Am J Med 1981;70(1):65-76

7 Brichet A, Ramon P, Marquette C. Post-intubation tracheal stenosis and ruptures. Reanimation 2002;11:1-10

8 Anand VK, Alemar G, Warren ET. Surgical considerations in tracheal stenosis. Laryngoscope 1992;102(3):237-243

9 Laccourreye, H, Pech, A, Piquet, JJ, et al. Laryngotracheal stenosis of adults and children. Arnette, Paris: Report of the French Society of ORL; 1985:29-155
10 Grillo HC, Donahue DM, Mathisen DJ, Wain JC, Wright CD. Postintubation tracheal stenosis. Treatment and results. J Thorac Cardiovasc Surg 1995;109(3):486-492, discussion 492-493

11 Keane WM, Denneny JC, Rowe LD, Atkins JP Jr. Complications of intubation. Ann Otol Rhinol Laryngol 1982;91(6 Pt 1) :584-587

12 Pontoppidan H, Geffin B, Lowestein E. Acute respiratory failure in adult. N Engl J Med 1972;287-799

13 Baugnée PE, Marquette $\mathrm{CH}$, Ramon P, Darras J, Wurtz A. Endoscopic treatment of post-intubation tracheal stenosis. A review of 58 cases. Rev Mal Respir 1995;12(6):585-592

14 Sinha R, Correia R, Gardner D, et al. Mucosal injury following short-term tracheal intubation: a novel animal model and composite tracheal injury score. Laryngoscope Investig Otolaryngol 2018;3(4):257-262

15 Rosow DE, Barbarite E. Review of adult laryngotracheal stenosis: pathogenesis, management, and outcomes. Curr Opin Otolaryngol Head Neck Surg 2016;24(6):489-493

16 Zribi S, et al. Acquired tracheal stenosis. J Tun Orl N 2009;22:48

17 Ferretti GR, Bricault I, Coulomb M. Helical CT with multiplanar and three-dimensional reconstruction of nonneoplastic abnormalities of the trachea. J Comput Assist Tomogr 2001;25(3):400-406

18 Madan K, Agarwal R, Aggarwal AN, Gupta D. Utility of rigid bronchoscopic dilatation and mitomycin $\mathrm{C}$ application in the management of postintubation tracheal stenosis: case series and systematic review of literature. J Bronchology Interv Pulmonol 2012;19(4):304-310

19 Kumar A, Asaf BB, Puri HV, Abdellateef A. Resection and anastomosis for benign tracheal stenosis: single institution experience of 18 cases. Lung India 2017;34(5):420-426

20 Yamamoto K, Kojima F, Tomiyama K, Nakamura T, Hayashino Y. Meta-analysis of therapeutic procedures for acquired subglottic stenosis in adults. Ann Thorac Surg 2011;91(6):1747-1753

21 Saloua O, Ismail N, Noureddine E, et al. Acquired laryngo-tracheal stenosis and its therapeutic difficulties. Research 2016;3:1639

22 Gaissert HA, Grillo HC, Wright CD, Donahue DM, Wain JC, Mathisen DJ. Complication of benign tracheobronchial strictures by self-expanding metal stents. J Thorac Cardiovasc Surg 2003;126(3):744-747

23 Froehlica P, Trey G, Stamm D, Florel D, Margen A. Role of long term stenting in management of subglottic stenosis. J Pediatr Otolaryngol 1993;27(3):273-280

24 Huang CJ. Use of the silicone T-tube to treat tracheal stenosis or tracheal injury. Ann Thorac Cardiovasc Surg 2001;7(4):192-196

25 Dass A, M Nagarkar N, K Singhal S, Verma H. Tracheal T-tube stent for laryngotracheal stenosis: ten year experience. Iran J Otorhinolaryngol 2014;26(74):37-42 